\title{
Systematisation of seismic retrofitting in vernacular architecture
}

\author{
A. Lima, M. R. Correia, F. Gomes, G. D. Carlos \& D. Viana \\ CI-ESG, Escola Superior Gallaecia, Portugal
}

P. B. Lourenço

ISISE, University of Minho, Guimarães, Portugal

\author{
H. Varum \\ University of Porto, Porto, Portugal
}

ABSTRACT: This paper aims to identify and to systematize the data obtained during the working process of the research project 'SEISMIC-V - Vernacular Seismic Culture in Portugal' in terms of seismic-retrofitting elements earthquake-resistant reinforcement implemented in vernacular buildings. The data will be distinguished based on the structural seismic strengthening and according to the following criteria: strengthening elements, perimeter seismic-resistant elements, arches reinforcing elements, and combined reinforcing elements.

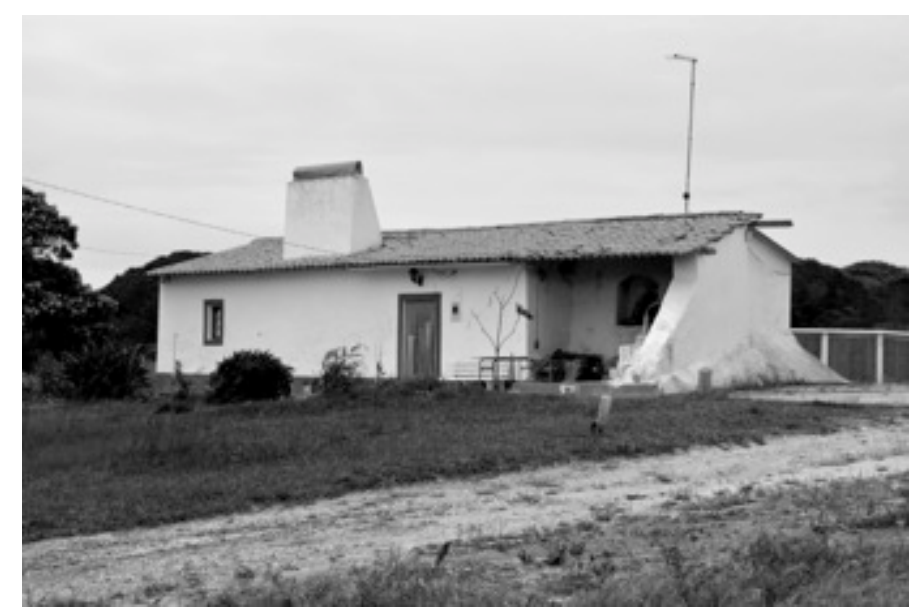

Figure 1. House of compact design with reinforcing elements, in Melides, Portugal (credits: CI-ESG).

\section{INTRODUCTION}

Although Portugal is recognised as a country of moderate seismic risk, the fact is that future occurrences of earthquakes are likely and damage will be important. The most significant earthquakes of the last century occurred between 1531 and 1998, causing considerable damage in various regions. The most affected regions have developed, in specific moments, seismic cultures based on the introduction of reinforcement elements and techniques. The constructive culture of each region generated different approaches, both in reaction to the damages arising from seismic events, and as prevention for future damage repetitions. These consisted simply in the integration of structural elements in the building methods and in the traditional materials, consequently enhancing the constructive resistance.

Through the empirical knowledge of local populations different approaches were put into action: the morphological and the tectonic one, which contributed to the improvement of traditional building systems, due to the effects of seismic events over the past centuries. On the basis of the morphological approach is the specification of the different typologies of simplified dwellings construction, and the consequential introduction of a typology of compact, uniform and homogeneous features. This typological simplification allowed a greater control of the response of the volume in a seismic event. On the other hand, the tectonic approach develops the earthquake-resistant reinforcement elements hosted throughout the different occurrences, both in new housing constructions, and in already existing constructions that required a greater structural stability.

The elements identified throughout the different work assignments were divided into four distinct groups, considering their structural usefulness:

a) Strengthening elements of traditional building techniques, including rammed-earth (most characteristic construction technique of the study area).

b) Perimeter seismic-resistant elements. The perimeter elements appear mostly linked to the rural typology and aim at define a joint action of the walls and floors (i.e. the so-called box behaviour), ensuring the perimeter stability of the volume.

c) Arches reinforcing elements. The strengthening system usually arises associated with arches located on the ground floor, of traditional buildings, 
in urban or periurban areas, with typologies of more than one floor.

d) Combined reinforcing elements. This group comprises the structural elements applied between contiguous or continuous buildings.

\section{MORPHOLOGICAL APPROACH}

\subsection{Simple and compact}

One the most relevant earthquake-resistant strategies to the structural stability of the dwelling is its simplicity. The more compact, uniform and homogeneous a house plan and elevation are, the more stable it will be. Thus, a uniform distribution of structural elements prevents the torsional effects of the volume. The type of isolated earthquake-resistant housing, identified throughout the study area, has a ground floor, in general presenting a longitudinal layout, with few internal partitions. The featured gable roof allows the evenly sharing of actions on the rammedearth (monolithic) or stone masonry bearing walls. A special concern with the relationship between the thickness and the height of the walls is notorious, so as to obtain adequate distribution of loading, to capacity of buttressing and to ensure its stability, in general. The lack of openings, typically a door and one or two windows, works towards the same end, as the greater the number of openings are, the larger is the area of structural vulnerability. The arrangement of the interior vertical walls demands for regular spacing in both directions, so as to provide bracing and a global structural behaviour.

Considering continuous housing (or compound arrangement, or semi-detached housing) this even distribution of forces was sought by symmetrical continuity of the volumes. The symmetry of the plan provides uniform distribution of the masses and stiffness, so as to avoid larger torsional effects. The longitudinal outer walls are braced by little spaced transverse walls, which function as partition walls. This arrangement allows the assembly to behave favourably and uniformly. The structure is simple, so that its response in case of a seismic event is able to distribute the forces evenly. When the row-housing plan adopts a T, L, or U shape, it is common that the inner walls are extended in both directions to prevent the different volumes to perform as isolated items. Similarly, protruding intersections may present buttresses in the corner, in order to prevent torsion and to avoid separation, points of special weakness for the stresses during an earthquake.

\section{TECTONIC APPROACH}

In regards to the tectonics of the housing, two types of actions taken by people can be distinguished: a reactive action, which attempts to mitigate the damage already produced by an earthquake on the building structure; and a preventive action, which aims to anticipate future events and limit damage. The analysed case studies indicate that it is not unusual to combine both actions in certain techniques and solutions. Moreover, after a seismic occurrence, people have adopted reactive and preventive techniques, simultaneously. This tectonic approach will be systematised in groups or "categories" of elements identified as earthquake-resistant. The first elements pointed out will be those used as reinforcing in traditional construction techniques; a second group will include those elements intended to tie together and provide safety to the system (box) of outer walls, ensuring the stability of the whole perimeter. Later on, a third group is composed of the buttressing elements that are responsible for strengthening the structural stability of the building; and finally, a fourth group will be set, which adopts structural elements of collaboration between contiguous buildings.

\subsection{Systems (elements) of structural reinforcement using traditional constructive techniques}

Rammed-earth is the most characteristic building system in the rural areas of our study area. One of the main reinforcements found at the level of the building system was the incorporation of horizontal reinforcement layers. Courses of tile, ceramic brick, stone or timber were observed among the monoliths blocks of rammed-earth, in order to, on the one hand, facilitate the transmission of bending action and inertia forces between the transverse walls (Fig. $2 a)$. For this purpose the horizontal ties should intersect each other, connecting perpendicular walls, and preventing the separation of the walls in the corner. On the other hand, these elements also prevent the spread of vertical cracks, which would inhibit the collapse of large wall sections or even entire walls. This same reinforcement was also identified in clay brick walls that had horizontal wooden elements irregularly spaced in the wall.

Another element to strengthen the structural stability of the building volume, also identified in the study area, is the relieving arch. Integrated into the vertical faces, on the lintels of doors and windows, these arches are responsible for diverting the vertical loads carried by the wall or roof on these fragile areas. They are usually constructed of solid brick or stone (Fig. 2B).

Finally, it is relevant to mention the 'pombalino' system, as a structural reinforcing system, built in. This system was used in the downtown of Lisbon and the downtown of Vila Real de Santo António, following the earthquake of 1755. 

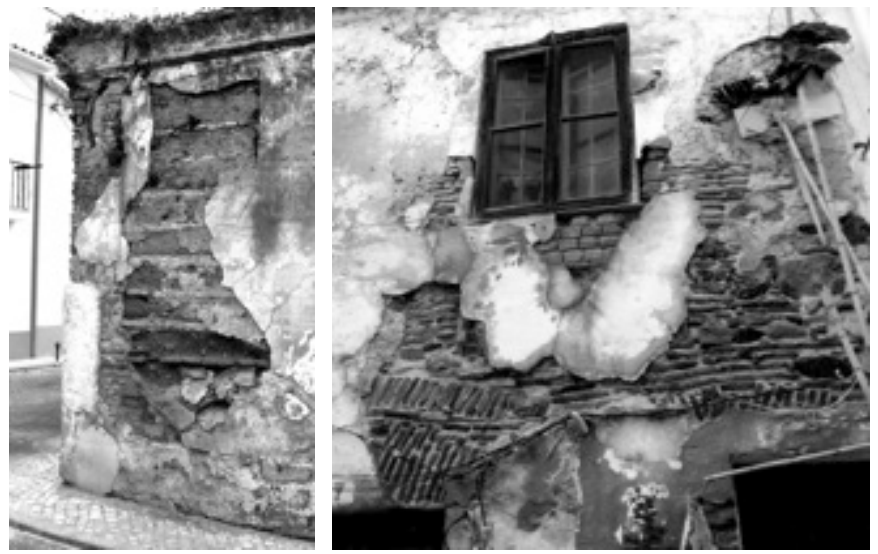

Figure 2. a) Horizontal rammed-earth reinforced with fired brick, Benavente. b) Relieving arches in Alcácer do Sal, Portugal (credits: CI-ESG).

\subsection{Perimeter seismic-resistant elements.}

The buttress (contraforte, gigante) is the most common seismic reinforcement element identified among the case studies. It is used, both as a reactive and as a preventive action. Also known as giants or fence posts, the buttress provide stiffness and additional support to the construction, and its main purpose is to consolidate the exterior walls. Usually in the form of a triangle, it is perpendicularly arranged to the outer facades.Within the surveyed cases, the buttress disposal, if not arbitrary, can vary substantially. In the edge walls it often appears centered, until the height of the eaves, while another, usually of smaller dimension, can be laid in the continuation of the longitudinal facade.

The walls of greater length, in which the openings generally locate, may also have a buttress, which is almost always located in the middle of the facade or leveled with the interior walls. Buttresses at the corner were also identified, in the connection between two perpendicular walls (areas of greater structural risk). These were found in sets of contiguous buildings of a significant dimension. The identified buttresses are usually built in regular masonry stone and solid brick. When the buttress is adopted as a reactive measure, and does not constitute an original element, it is common to observe the lack of cohesion to the backed wall. Another element that adds to the structural stability of the building is the strengthening of the corners. The corners of the buildings, and likewise of the houses built in continuous row, are extremely weak points, being especially sensitive to the action of the two directions of the earthquakes. The corners should ensure the connection between the transverse walls, being therefore exposed to large forces. Identified in areas of urban and semiurban setting, the strengthening of the corner arises on the enclosure walls, in a trapezoidal shape, up to of the first floor. It is defined as a proliferation of the corner section. It also works as a buttress at the corner, gathering the volume lengthwise and crosswise.

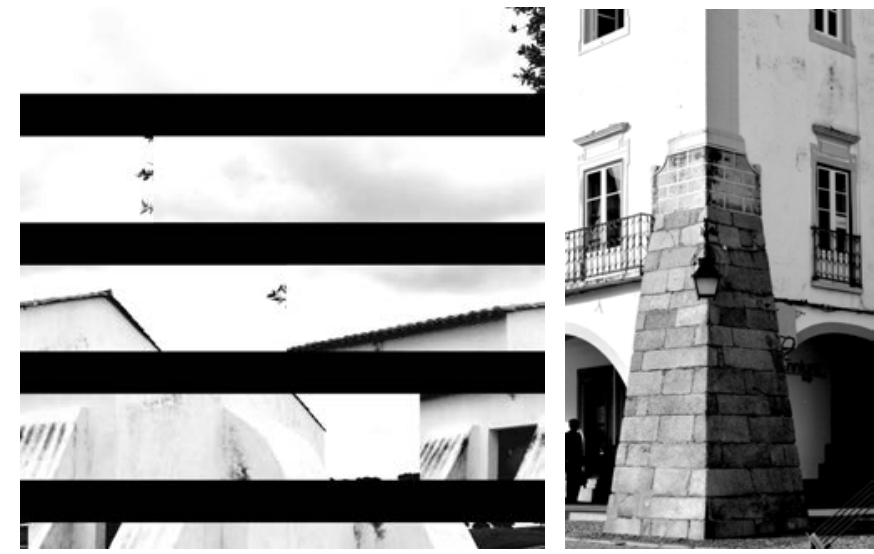

Figure 3. a) Reinforcement Buttresses, Baleizão. b) Corner reinforcement, Évora. (credits: CI-ESG).

In Alentejo, it is common to have fired brick or stone masonry plinths, above the ground or floor level, due to the high capillarity arising from the soil. In some cases, it was detected an addition of masonry at the building's plinth, extending the wall width. This was for instance the case of some buildings in Evora historical center. The reinforced plinth helps spread the loads from the above walls and bring additional strength and stability to the building.

In Alentejo and Ribatejo housing typology it is common to observe the 'poial', an element, usually built in stone, near the outer wall, which in addition to helping its structural consolidation, it is also used as a bench. Throughout the analysed case studies, the masonry plinth acquires some relevance, being identified in Melides, Benavente, Évora, S. Brissos, Lagos and the Azores.

In the case of Melides, the poial has an orthogonal section, and occupies the space between the top of the facade buttresses, as a structural consolidation complement.

In S. Brissos both models were identified: plinths of trapezoidal form, consolidating the gable at the edges of the built volume; and the poial reinforcing more the outer walls.

In Benavente, the plinth arises as a reinforcement element of the building set, closing the block in Ushaped, and consolidating the side gables. The plinth is also one of the most important elements in the Azores, where numerous case studies were identified. It is usual for the plinth to take full perimeter of the housing, thus resisting the longitudinal and transverse forces, and acting as an outer reinforcement ring.

The tie-rods are steel rods intersecting two opposite outer walls, to the height of the eave. These rods are anchored in the outer faces of the walls by metallic anchoring devices, confining the structure in the two directions. Cases were identified where this solution was applied in both the longitudinal and in transverse direction, thus connecting the four façades of the building. 

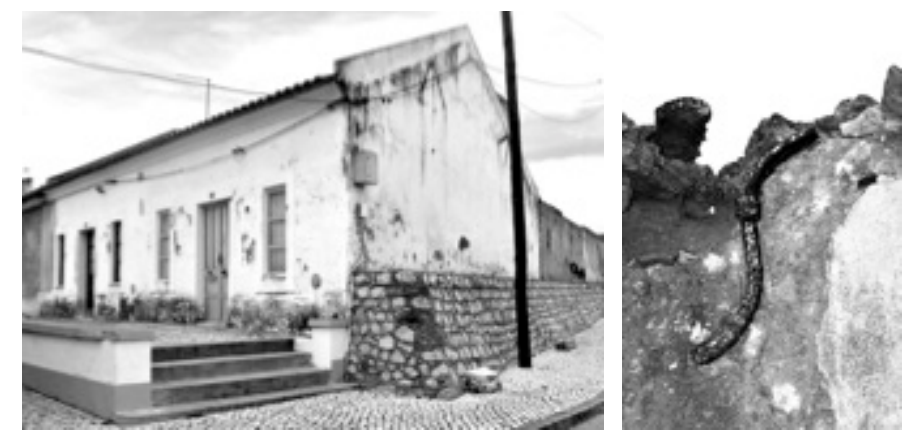

Figure 4. a) House in Benavente, with tie rods and plinth as reinforcements. b) Metallic anchoring device (cat), Melides. (Credits: CI-ESG).

Along with the buttress, the tie-rod is the structural reinforcing member with more presence within the study area. Tie-rods were identified in Melides, Trindade, Alcacer do Sal, Coruche, Benavente, Baleizão, Tavira and Lagos. The tie rod appears as a reinforcement element, both in the preventive, and in the reactive approach. In cases, such as those identified in Melides, it is clearly adopted as a reactive strengthening solution, subsequent to a seismic occurrence, to reinforce a pre-existing structure.

In Benavente, the tie-rods placed next to the cover, or between the floors structures, when they are between floors, have been adopted as a preventive measure in the reconstruction and expansion of the urban structure after the 1909's earthquake.

Considering Melides, at the height of the tie rods were also identified continuous cornices, aiming at the increasing its resistance. These elements incorporate steel rods joined with a connector placed after the removal of the plaster along the contour of the housing, fixing the existing structural damage.

\subsection{Arches reinforcing elements.}

Considering urban or suburban typologies with more than one floor, the vertical forces of the building and of the entire set are transmitted through main (or primary) walls and pillars, which are connected either by arches, or arches and vaults. These solutions confer more resistance to the base of the building in a seismic occurrence, as forces are distributed more evenly.

The vaults that cover the ground floors are, generally, quadripartite vaults, consisting of four bent surfaces intersected by diagonal protruding ribs. The perimeters of the vaults encompass transverse arches that, in addition to the underpinning of the vaults, they also help to convey their loads to the walls and pillars (Fig 5.a).

Another arch-reinforced element identified in the study area is the flat arch or jack arch. These are
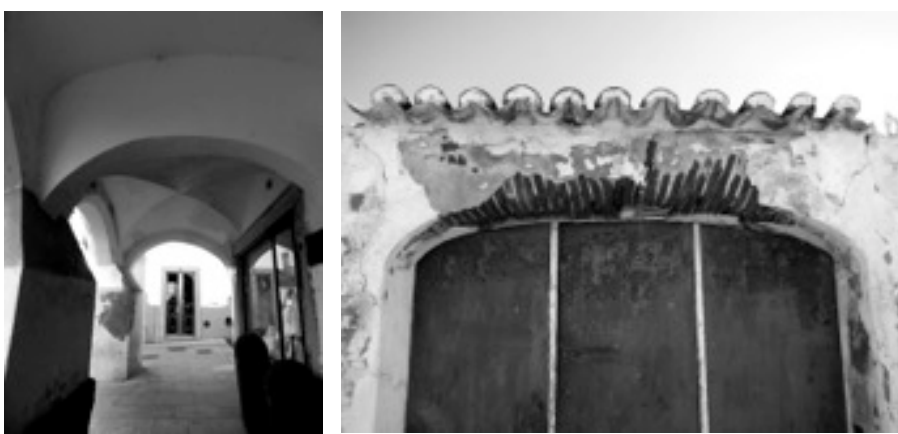

Figure 5. a) Arcades and vaults system in the historical centre of Évora. b) Flat arch or Jack Arch, Castro Marim (Credits: CI-ESG).

elements that replace lintels, in order to achieve greater flexible resistance to the span set. The arches transfer vertical loads, distributing them laterally from the upper walls. Typically, they are constructed in fired brick, and take the entire thickness of the masonry (see Fig. 5.b).

\subsection{Combined reinforcement elements.}

The counter-arch is a structural element intended to block the breakdown mechanisms between adjoining buildings, typically of different heights. Its main function is to redistribute the horizontal movements at the floor level to the vertical walls of the nearby buildings. It is a kind of structural cooperation between adjacent volume structures, so that the collapse of one wall, does not foster a similar action in the adjacent buildings. For years it was assumed as a resource, widely used to consolidate structures that had been damaged, and where the collapse mechanism had already begun. Within this study area this element has been identified mainly in the Central Alentejo region, particularly in the area of Evora, as a structural reinforcement of cooperation between adjacent buildings, in order to ensure its entire blockade and a uniform behaviour towards an earthquake, in order to avoid the consecutive collapse of the built structure. (Fig.6).

The continuous cornice (cimalha) appears as tying element between different units. This element allows the upper trim of the facade, functioning as a cross lintel to different walls in the same alignment. In addition to the vertical strengthening building system, it also allows the reception of the roof structure, either in the transverse or supporting the eaves framework. These elements generally comprise solid brick masonry or stone masonry, forming a sharp protrusion in contact with the roof eaves. In certain examples it may also be constituted by metal straps. When in stonework, they are usually left at sight, and when built with brick they are usually plastered and painted like the façade. 


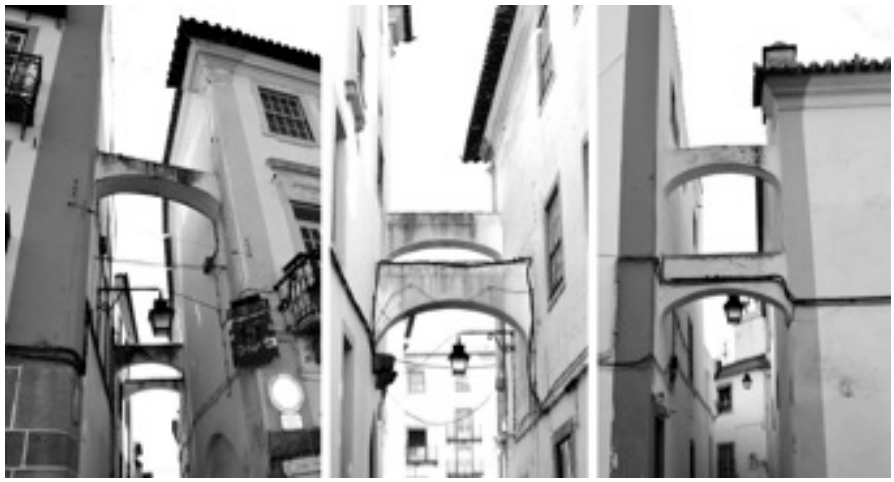

Figure 6. Different reinforcing counter-arches in the historical centre of Évora (credits: CI-ESG).

As well as ensuring a better horizontal leveling of the distribution of the load of the covering, and thus ensuring greater dispersion of forces in the volume, it is usually associated with a systematisation of arch centering and alignments of roof coverings. Thus, it also contributes to the dismissal of dissonant elements in the general volume. The continuous cornice is intended to neutralise the breakdown of contiguous walls, avoiding cracking at their contact edges, as well as the counter arch. It also prevents the consequent action of 'hammering' (pounding), capable of occurring in adjacent structures (Fig.7).

\section{FINAL REMARKS}

Considering the observation and analysis of the collected data, a series of structural reinforcing elements used in the past as a response to a seismic event are identified. These are elements and techniques that are applied to reach increasing safety. Its implementation is directly linked to the intensity and the frequency of the occurrence, and it is not systematic, but occurs recurrently. The elements and techniques identified are, generally, common to the different studied areas. They can be distinguished as systems implemented, almost exclusively, in singlefamily housing, of isolated character; and also elements of a more urban character (application). Likewise, all systems, urban and peri-urban, and rural, can be classified according to its structural function.

Through the analysis carried out it is possible to conclude that, at an urban context implementation level, the elements and techniques applied point out more to the reinforcement of the set, than to the individual housing unit. The only elements identified with an isolated function were the tie rods. Most of the data collected point to structural cooperative systems between building blocks or contiguous dwellings: plinth at the base transversal to a set of buildings, the counter arch as a liaison (structural cohesion) between adjacent blocks, and

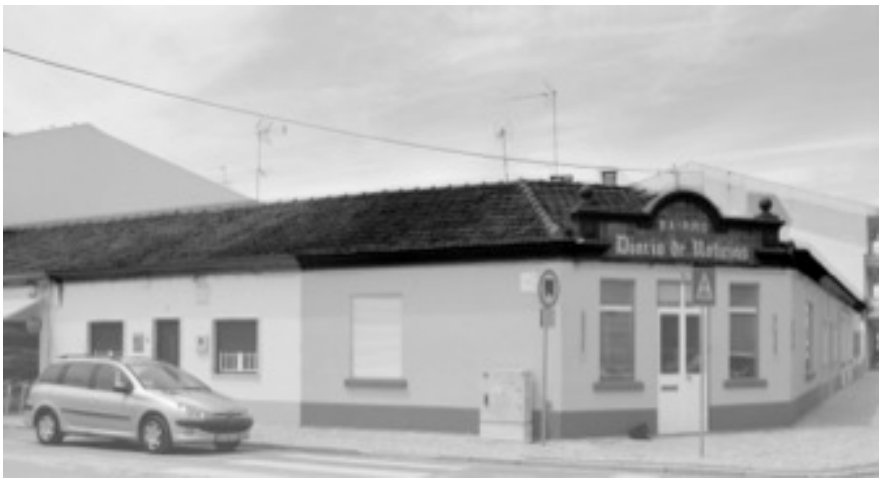

Figure 7. Continuous cornice in Bairro das Noticias, Benavente. (Credits: CI-ESG).

homogeneous structural systems, such as arcades and vaults, or continuous cornice. In case of the isolated housing, the results point to the collaboration between different systems and strengthening techniques into the unit, as the buttresses, the tie rods, the poial, the masonry vaulted structure at the base, and the strengthening of the traditional building techniques.

The analysis can also synthesize a classification of the elements and techniques according to its structural function: perimeter reinforcing elements, cooperative reinforcing elements, reinforcing elements of from the constructive technique and arch-reinforcing elements. The results also reveal a greater integration of the perimeter reinforcing elements in the rural context than in the urban context. Moreover, when these elements are identified in any urban context, these always work as a structural element of cooperation between dwellings or blocks, and rarely in isolation. The cooperative reinforcing elements were so linked with the urban environment systems of a set.

The arch-reinforcing elements were found in both contexts, in rural areas as spans reinforcement system, and in urban context as structural continuous systems. The strengthening traditional building systems are also identified both in rural and urban areas.

\section{ACKNOWLEDGMENT}

The authors gratefully acknowledge the support by the Portuguese Science and Technology Foundation (FCT) to the research project 'SEISMIC-V - Vernacular Seismic Culture in Portugal' (PTDC/ATPAQI/ 3934/2012).

\section{REFERENCES}

Correia M., Gomes, F. \& G., Duarte Carlos, G. (2014). Projecto de Investigação Seismic-V: Reconhecimento da Cultura Sísmica Local em Portugal in Arquitectura de Tierra: Patrimonio y Sustentabilidade en regiones sísmicas, eds. M. Correia, C. Neves, R. Núñez, El Salvador, Imprimais. 